\title{
Impacts of a delayed and slow-paced vaccination on cases and deaths during the COVID-19 pandemic: a modelling study
}

Gustavo Libotte ( $\nabla$ glibotte@lncc.br)

National Laboratory for Scientific Computing https://orcid.org/0000-0002-4583-6026

Lucas Anjos

National Laboratory for Scientific Computing https://orcid.org/0000-0002-9623-5570

Regina Célia Cerqueira de Almeida

National Laboratory for Scientific Computing

Sandra Mara Malta

National Laboratory for Scientific Computing https://orcid.org/0000-0003-1272-6039

Roberto Medronho

Federal University of Rio de Janeiro https://orcid.org/0000-0003-4073-3930

\section{Research Article}

Keywords: low vaccination coverage, delayed start of vaccination, resurgence of cases, avertable deaths, COVID-19

Posted Date: November 17th, 2021

DOI: https://doi.org/10.21203/rs.3.rs-936335/v2

License: (9) (1) This work is licensed under a Creative Commons Attribution 4.0 International License. Read Full License 


\section{Research in context}

\section{Evidence before this study}

Although vaccination rates against COVID-19 have increased over time, part of the population still harbours suspicions about the vaccine's benefits. Misinformation, shaped to a great extent by political and religious ideologies, makes many people unwilling to get vaccinated. Lack of effort and strategic planning caused delays in the availability of vaccines, also making the vaccination process often lethargic. Furthermore, many people have also neglected the second dose of vaccines, not achieving the expected protection. As these are global concerns, there is an immediate need to obtain answers on how these factors affect the mitigation of COVID-19. In particular, all these factors have been affecting the progress of vaccination against COVID-19 in Brazil. We searched PubMed up to October 27, 2021, with no language restrictions, using the search term "((avertable deaths) OR (delayed start of vaccination) OR (low vaccination coverage) OR (resurgence of cases)) AND (COVID-19) AND (vaccines)." We found only nine studies that also analysed some impacts of delayed or slow-paced vaccination on the COVID-19 pandemic, inferring the number of possible cases and deaths resulting from low vaccination coverage. However, to our knowledge, no modelling analysis has sought to comprehensively study all of these factors and how they affect the number of infected and dead individuals in a population.

\section{Added value of this study}

This study provides a quantitative insight of an intuitive perception of global concern, the damage caused by a vaccination campaign that is sometimes negligent and inadequate, in the face of a pandemic. Since we consider achievable scenarios, from the point of view of the start date of vaccination and the efforts to increase vaccination rates and achieve greater vaccination coverage (based on the evidence before this study), the comparison with the factual situation expresses how such efforts could have mitigated the consequences of the pandemic. Estimating such consequences in terms of the number of dead and infected individuals is a pragmatic line of reasoning for analysing how bad decisions can affect the population as a whole. The added value of this study is also related to the computational framework proposed to conduct the analyses, which can be used in supplementary studies.

\section{Implications of all the available evidence}

An immediate implication of this study is to give support for driving the prioritisation of measures to enhance COVID-19 vaccination campaigns around the world, given the impacts that were quantified in this analysis. Furthermore, the eventual emergence of possibly more lethal variants, the antagonism between limited access to vaccines and their acceptance, and seasonality protection against the virus demand continuous immunisation supply chain improvement. 


\title{
Impacts of a delayed and slow-paced vaccination on cases and deaths during the COVID-19 pandemic: a modelling study
}

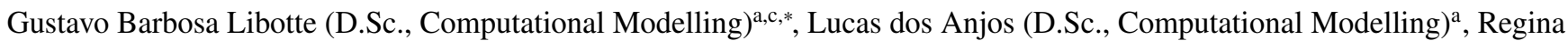
Célia Cerqueira de Almeida (D.Sc., Nuclear Engineering) ${ }^{\mathrm{a}, \mathrm{c}}$, Sandra Mara Cardoso Malta (D.Sc., Mathematics) ${ }^{\mathrm{a}}$, Roberto de Andrade Medronho (D.Sc., Public Health) $)^{\mathrm{b}, \mathrm{c}}$

\author{
${ }^{a}$ National Laboratory for Scientific Computing, 333, Getúio Vargas Av., Quitandinha, Petrópolis, 25651-075, Rio de Janeiro, Brazil \\ ${ }^{b}$ Department of Epidemiology and Public Health, School of Medicine, Federal University of Rio de Janeiro, 550, Pedro Calmon Av., Rio de \\ Janeiro, 21941-901, Rio de Janeiro, Brazil \\ ${ }^{c}$ Center of Excellence in Digital Transformation and Artificial Intelligence of the State of Rio de Janeiro (Hub.Rio): Thematic Network in the Fight Against \\ COVID-19, Federal University of Rio de Janeiro, 550, Pedro Calmon Av., Rio de Janeiro, 21941-901, Rio de Janeiro, Brazil
}

\begin{abstract}
Background: In Brazil, vaccination has always been cutting across party political and ideological lines, which have delayed its start and brought the whole process into disrepute. Such divergences put the immunisation of the population in the background and create additional hurdles beyond the pandemic, mistrust and scepticism over vaccines.
\end{abstract}

Methods: We conduct a mathematical modelling study to analyse the impacts of late vaccination and with slowly increasing coverage, as well as how harmful it would be if part of the population refused to get vaccinated or missed the second dose. We analyse data from confirmed cases, deaths caused by COVID-19, and vaccination in the state of Rio de Janeiro in the period between March 10, 2020, and October 27, 2021. The classical SIR model is extended to consider the effect of vaccination (efficacy, interval between doses, and vaccination rate) and data sets are regularised using Gaussian Process Regression. The model parameter distributions are estimated using Bayesian inference, aiming to obtain credible intervals in the simulations.

Findings: We estimate that if the start of vaccination had been 30 days earlier, combined with efforts to drive vaccination rates up, 31,657 $(25,801-35,117)$ deaths could have been averted. Our results also indicate that the slow pace of vaccination and the low demand for the second dose could cause a resurgence of cases as early as 2022.

Interpretation: The government's inaction and lack of a strategic plan to fight the pandemic meant that vaccination started late, leading to thousands of deaths that could have been prevented. Even when reaching the expected vaccination coverage for the first dose, it is still challenging to increase adherence to the second dose and maintain a high vaccination rate to avoid new outbreaks.

Funding: Carlos Chagas Filho Foundation for Supporting Research in the State of Rio de Janeiro (FAPERJ) and Brazilian National Council for Scientific and Technological Development (CNPq).

310 words

Keywords: low vaccination coverage, delayed start of vaccination, resurgence of cases, avertable deaths, COVID-19

\section{Introduction}

As of February 25, 2020, when the first case of infection with SARS-CoV-2 (severe acute respiratory syndrome coronavirus 2) was reported in Brazil, the country has accumulated more than 21.8 million confirmed cases and, on November 17, 2021, Brazil's death toll topped 610,000. Despite the critical situation to contain the ensuing epidemic and the resurgence of cases (especially with the emergence of new variants), Brazil had delays

\footnotetext{
${ }^{*}$ Corresponding author

E-mail address: glibotte@1ncc.br

Phone number: +55 22 98815-0034
}

in starting the vaccination campaign, compared to other countries, ${ }^{1}$ which took place on January 17, 2021.

Although Brazilians' tendency towards vaccination compliance is relatively high, ${ }^{2}$ some factors were partly responsible for the slowness of the mass vaccination campaign. The country is paying a price for the slow pursuit of vaccines early on, especially regarding the federal government's rejection of vaccines from Pfizer in mid-2020, ${ }^{3}$ in addition to the rebuke of the agreement signed with Sinovac; ${ }^{4}$ millions of people are also missing their second dose, especially because of misinformation, assuming that just one dose provides the expected immunity; ${ }^{5,6}$ temporary interruptions of vaccination services, due to a lack of shots, logistical problems or absence of supplies (particularly 
active pharmaceutical ingredient) $;^{7,8}$ furthermore, there are on the one hand people who try to jump the queue to get vaccinated early, and on the other hand those who choose not to get vaccinated, seemingly motivated by political ideology. ${ }^{9}$

The epidemiological situation in some states is particularly worrisome due to the level of government intervention, investments in health, the pace of vaccination, and population mobility. ${ }^{10}$ Political polarisation and the spread of fake news also hamper the fight against COVID-19 and the adoption of nonpharmaceutical interventions. ${ }^{11}$

Therefore, it is essential to investigate the likely consequences of such events and circumstances regarding the burden of the epidemic. For this purpose, we conduct this study aiming at investigating the following issues:

- What would be the influence of bringing forward or delaying the vaccination roll-out?

- How effective would a faster vaccination process be in mitigating the epidemic?

- How many deaths could have been averted if there had been more efforts to obtain and manage vaccines?

- How harmful is the choice of part of the population for not getting vaccinated?

- What is the effect of not taking the second dose of the vaccine on the population?

In this context, the objective of this work is to provide an analysis of scenarios related to the epidemic in Rio de Janeiro (RJ), one of the most important states in Brazil in terms of demographic density and economic relevance, to answer the issues raised employing computational simulations whose results can be compared to the current situation of the epidemic in the state. The general framework we propose can be extended to analyse the situation of the epidemic in any region.

\section{Methods}

552 words

\section{Model description}

We extend the well-known SIR model to incorporate the effects of vaccination in the population. The incidence of new cases is given by $\beta(t) S(t) I(t) / N$, where $N$ is the population size, assumed constant for simplicity (or due to the analysis time frame). Both susceptible and infected individuals are vaccinated at a rate proportional to $v$. Once infected, individuals are moved to the removed class at a rate proportional to $\gamma$, whereas the gain in the dead class is at a rate proportional to $\rho$. Vaccinated individuals can either be moved into the removed class or become susceptible again, respecting the interval between doses, $1 / \tau$, when applicable. In the first case, immunised individuals are moved into the removed class proportionally to $\eta$, the overall vaccine efficacy. In the second case, the susceptible class is fed back proportionally to $(1-\eta)$. The portion of individuals who do not receive the second dose is equal to $\alpha$ and we assume an overall impaired efficacy, given by $\bar{\eta}$. In general, the model admits $n$ classes of vaccinated individuals, depending on the types of vaccines used. The schematic representation of the model is shown in figure 1 .

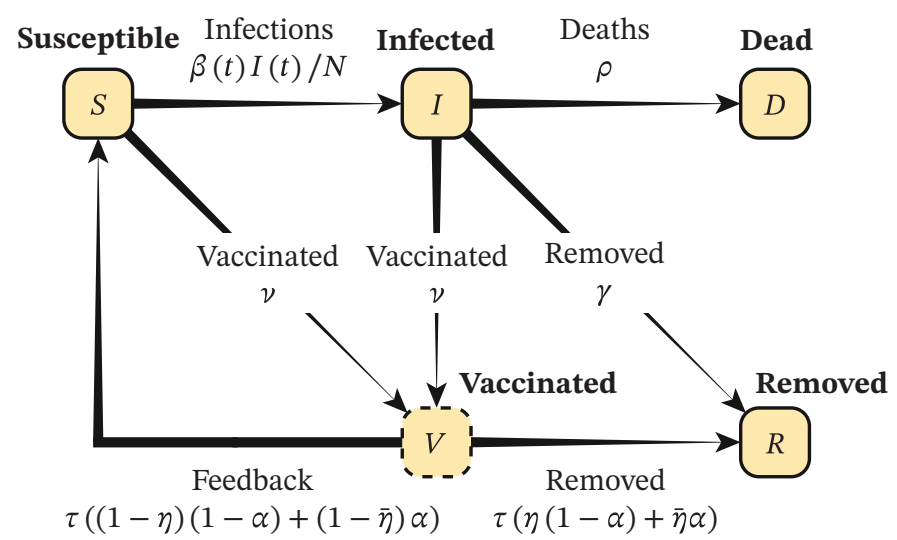

Figure 1: Schematic representation of the model.

\section{Case incidence and vaccination data}

Daily data on confirmed cases and dead individuals due to COVID-19 in RJ are divided into two subsets, from before and during vaccination. The former, considered the training set, ranges between March 10, 2020, the first day with at least five cases diagnosed, and January 19, 2021, the last day before the start of vaccination. The latter subset contains the data between January 20, the day the vaccination started, and October 27, 2021. Cumulative data on individuals vaccinated with the first dose and immunised (with both doses and with the single-dose vaccine) are also adopted from the same period. These data are provided by the Ministry of Health public repository ${ }^{12}$ that does not distinguish the type of vaccine used although there is information on the distribution of different vaccines for each Brazilian state.

\section{Data regularisation and inference of model parameters}

Daily data on infected and dead individuals in RJ are very noisy (refer to the Supplementary Material). ${ }^{13}$ In these circumstances, the regularisation of data emerges as an alternative to reduce the noise level, without misrepresenting data behaviour, to streamline the task of fitting model responses to the data set. In particular, Gaussian Process models are a probabilistic approach to representing arbitrary functions by means of a probability distribution over all possible functions that fit a set of points. ${ }^{14}$ Here we employ the mean of the posterior Gaussian 
Process Regression as the regularised data, which are used to estimate the model parameters.

Model outcomes are fitted to the training set using Bayesian inference. The parameters to be estimated are $\beta_{1}, \ldots, \beta_{4}$, which refer to the free parameters of the functional form of the time-varying transmission rate that we adopt, a sum of exponential functions capable of modelling transmission over multiple waves. We employ the Transitional Markov Chain Monte Carlo ${ }^{15}$ method for Bayesian model updating, sampling around predictive solutions previously obtained by means of least squares. $95 \%$ equal tailed credible intervals are obtained by excluding $2.5 \%$ from each tail of the posterior distribution. In all numerical results, the $95 \%$ credible intervals are shown in parentheses, following the result corresponding to the maximum a posterior.

\section{Results}

2,006 words

We consider that the target of individuals to be immunised in RJ is proportional to $80 \%$, which also corresponds to the number of inhabitants aged 12 years or over. ${ }^{16}$ The vaccination process is carried out with four vaccines, and the vaccination rates of each one is proportional to the number of doses granted to RJ by the Ministry of Health, as shown in figure 2A. The simulations are conducted considering three scenarios related to the overall vaccination rate: the base scenario is associated with the average vaccination rate at the time of writing this paper, that is, $v=0.40 \%$ of the population vaccinated per day. This corresponds to approximately 69,200 vaccinated individuals per day, which agrees with the average of daily vaccinations. In the two other hypothetical scenarios, we consider $v=0.35 \%$ and $v=0.50 \%$ of the population vaccinated per day. In this setting, approximately 60,550 and 86,500 individuals are vaccinated per day, on average, respectively. Figure $2 \mathrm{~B}$ shows the frequencies of vaccination rates considering both shots (single-dose vaccines count as second doses), given the cumulative number of individuals vaccinated per day, which in turn is shown in figure $2 \mathrm{C}$. The target vaccination coverage for the first dose would be reached in approximately 290 days, which means that $80 \%$ of the population would have received at least the first dose by November 2021, as supported by the prediction shown in figure $2 \mathrm{C}$. As for the second dose, the prediction indicates that the population would be immunised in the first months of 2022 , respecting the interval between doses.

\section{Benefits and risks regarding the pace of vaccination}

The influence of the pace of vaccination on the mitigation of the epidemic, in the matter of reducing the number of infected and dead individuals over time, is shown in figure 2D. Note that the vaccination data agree with the simulations, even if they were not used to estimate the model parameters. The same goes for the cumulative data from infected and dead individuals, indicating that the choice of model parameters seems to correspond to the actual epidemic scenario in RJ. Such simulations indicate that if the vaccination process were faster, allowing to vaccinate approximately 8,650 more people per day (with $v=0.5 \%$ ) compared to the amount vaccinated in the base scenario, on average, the number of cases could be reduced by $26.58 \%$, from $1,378,382(1,226,446-1,472,044)$ to $1,011,948$ $(944,239-1,054,368)$ cases, whereas the death toll would drop from $70,846(63,037-75,659)$ to $52,013(48,532-54,193)$. On the other hand, when the pace of vaccination is delayed proportionally to $v=0.35 \%$, the adverse effect is disproportionately greater: the number of confirmed cases would rise to $1,922,585$ $(1,607,030-2,128,694)$, a meaningful increase of $39.48 \%$, and deaths could reach 98,812 (82,596-109,401).

How the timing of vaccination roll-out affects disease mitigation

Here, we propose hypothetical scenarios in which the vaccination efforts get underway 10, 20 or 30 days before or after January 20, 2021. For each particular vaccination rate, we simulate the model for all combinations of proposed scenarios, whose outcomes are shown in figure 3A, concerning the daily number of infected and dead individuals. For an arbitrary vaccination rate, it is clear that starting the vaccination campaign a few days earlier is beneficial both in terms of "flattening the curves" and in terms of suppressing the epidemic. Take as an example the scenario in which $v=0.40 \%$. On June 4, 2021, when simulations show that the daily death toll would peak if vaccination had started 30 days late, there would have been 893 deaths $(700-1,001)$. On the same day, had the start of vaccination been 30 days early, there could have been only 34 deaths (28-38). Note that in the latter case, deaths would peak on June 5, 2020 (first wave), at 148 deaths (146-151).

Figure 3B shows how delaying the start of vaccination combined with vaccination at a slow pace could be devastating to the population. In the worst-case scenario, with vaccination coverage increasing slowly $(v=0.35 \%)$ and vaccination campaign starting 30 days after January 20, 2021, the number of infected individuals could have reached 6,398,467 (4,754,038$7,462,813)$, whereas there could have been 328,781 deaths $(244,308-383,445)$. If we look at the opposite scenario, when more effort is put into a rapid vaccination $(v=0.50 \%)$ that has started 30 days before the actual day, the number of cases and deaths would drop to $660,212(637,960-677,854)$ and 33,935 (32,791-34,841), respectively. The numerical results for all analysed scenarios are shown in table 1 .

We also sought to directly relate the number of vaccinated and dead individuals, aiming to analyse the likely hardship to the population when the start of vaccination is delayed, compared to the scenario in which vaccination had started earlier. Suppose vaccination had started on February 19, 2021, 30 days beyond the actual date, when 313 deaths (291-324) would have been confirmed, as shown in figure $3 \mathrm{C}$. Based on the benchmark vaccination rate, the simulations show that the deaths would 

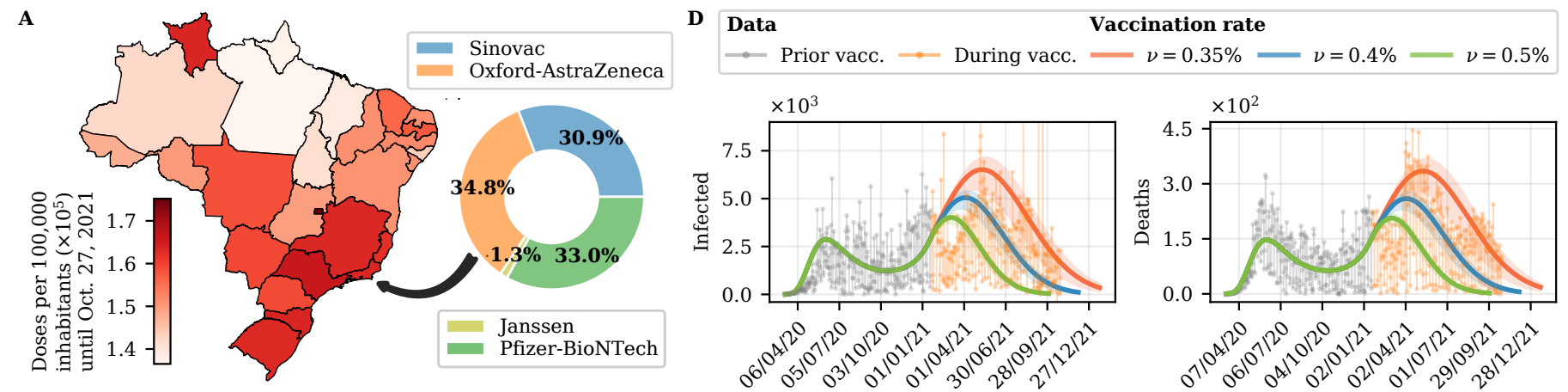

Vaccination rate
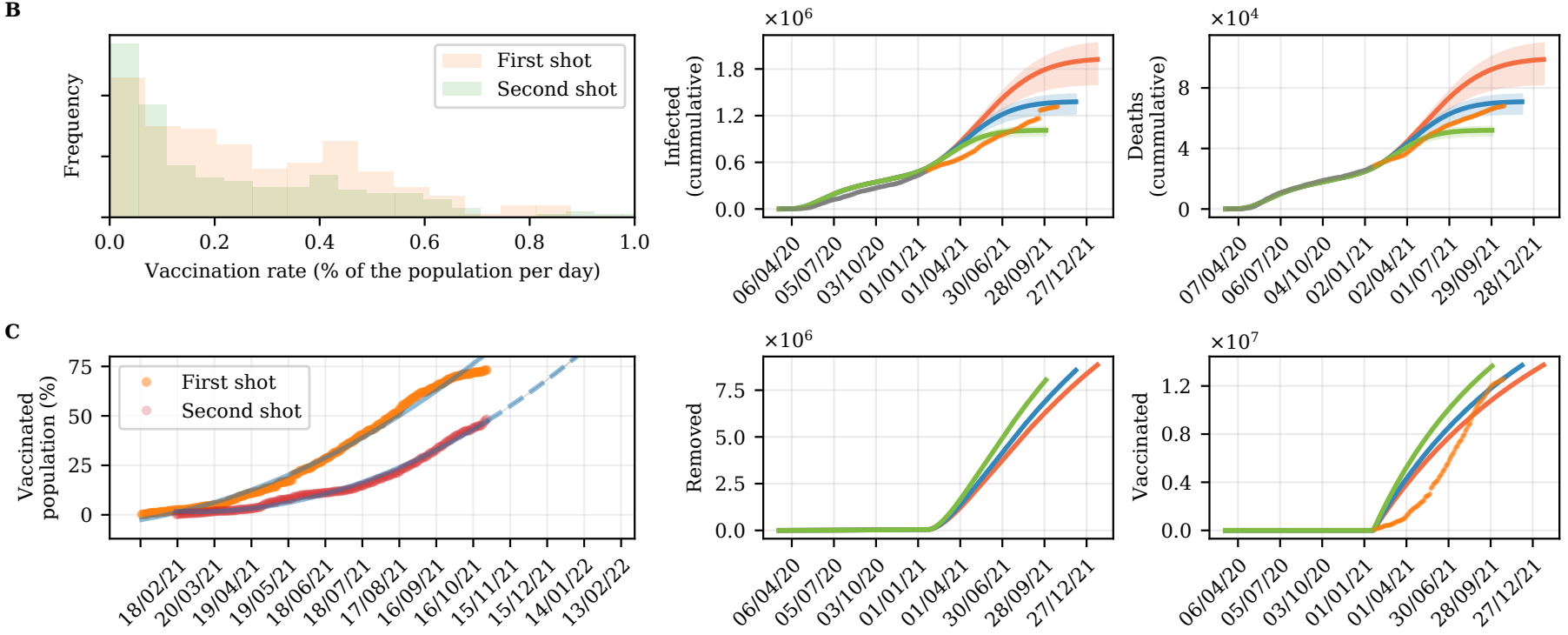

Figure 2: Speed in vaccination and expectation of disease mitigation in RJ. (A) Map of vaccine doses distributed in each Brazilian state per 100,000 inhabitants, and the portion of each type of vaccine destined for RJ, until October 27, 2021. (B) Frequency of vaccination rate for each shot, in terms of percentage of population per day. (C) Perspective of coverage of the population eligible for vaccination for each shot. (D) Model simulation considering different vaccination rates, slow $(v=0.35 \%)$, intermediate $(v=0.40 \%)$, and fast $(v=0.50 \%)$, taking into account the frequencies (B) and the proportion of vaccines of each type (A). The model is simulated until reaching the same amount of vaccines administered in each scenario (see the lower right frame). The shaded areas represent the $95 \%$ credible interval. Note that the period of the end of vaccination in the scenario where an intermediate vaccination rate is adopted agrees with the prognosis in (C).

peak approximately in June 2021, at 893 deaths (700-1001). At this time, about $6,028,386$ people $(6,026,531-6,032,208)$ could have been vaccinated (with both doses and with the single-dose vaccine), representing approximately $34.84 \%$ of the population. However, the worst-case scenario would bring out a far more ruthless possibility: even with approximately 7,081,206 people immunised $(7,078,053-7,091,406)$, deaths would peak in July 2021 , reaching 1,405 deaths in a single day $(1,010-1,640)$. This means that, despite having been vaccinated nearly $17.46 \%$ more people, comparing both scenarios, a record-high daily death toll could have been reached, to a great extent driven by the late start of vaccination.

According to the simulations, in 2020 the effective reproduction number (see the Supplementary Material) was only below the threshold $\mathcal{R}(t)=1$ between June and October, as shown in figure $3 \mathrm{D}$. Despite that, in this period the lowest value reached was $\mathcal{R}(t)=0.912$, at the end of July. Afterwards, the effective reproduction number was always above one, until the vaccination started to take effect. At this point, assume that the vaccination had been brought forward by 30 days. On the same day as the start of vaccination, 1,834 new cases $(1,786-1,862)$ would have been confirmed. Even maintaining a slow pace of immunisation $(v=0.35 \%)$, the transmission potential of SARS-CoV-2 could have reached $\mathcal{R}(t)=1$ as early as February 2021 (approximately three months after the hypothetical start of vaccination), when RJ would have vaccinated $24.19 \%$ of the eligible portion of the population.

\section{Potential aftermath of COVID-19 vaccine hesitancy}

We now simulate the model considering only $70 \%$ vaccination coverage, a reduced amount due to people who are unwilling to be vaccinated. Figure $4 \mathrm{~A}$ shows the model outcomes for the daily number of infected and dead individuals over time, given the three vaccination rates we have assumed, alongside the cumulative number of vaccinations. Considering the benchmark vaccination rate, $70 \%$ vaccination coverage would be reached 
A
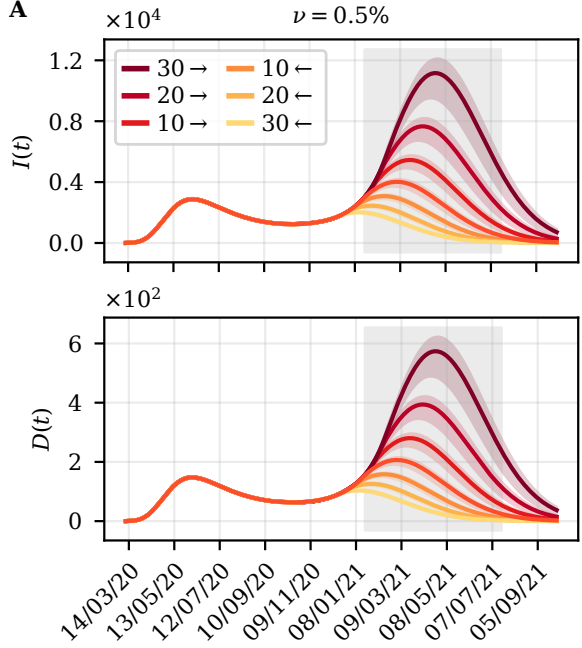

B

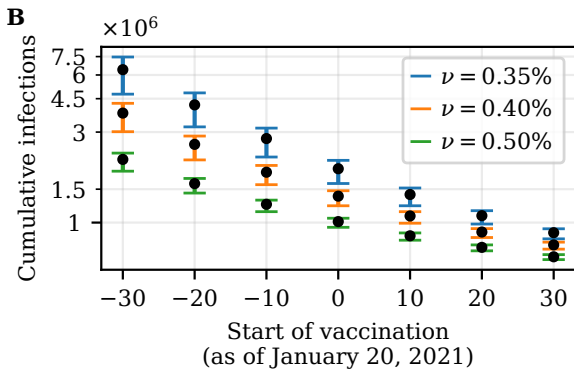

C

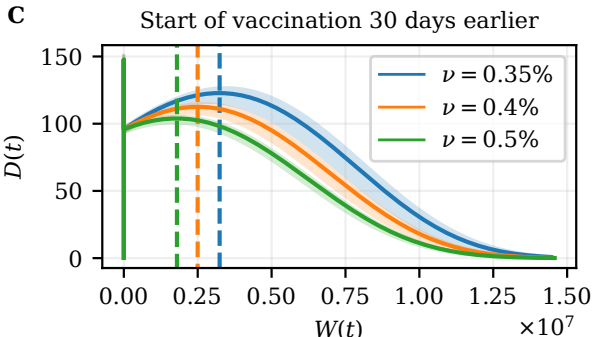

Approximate period of peak occurrence - $=01 / 2021 \quad-=01 / 2021 \quad-\quad 03 / 2021$
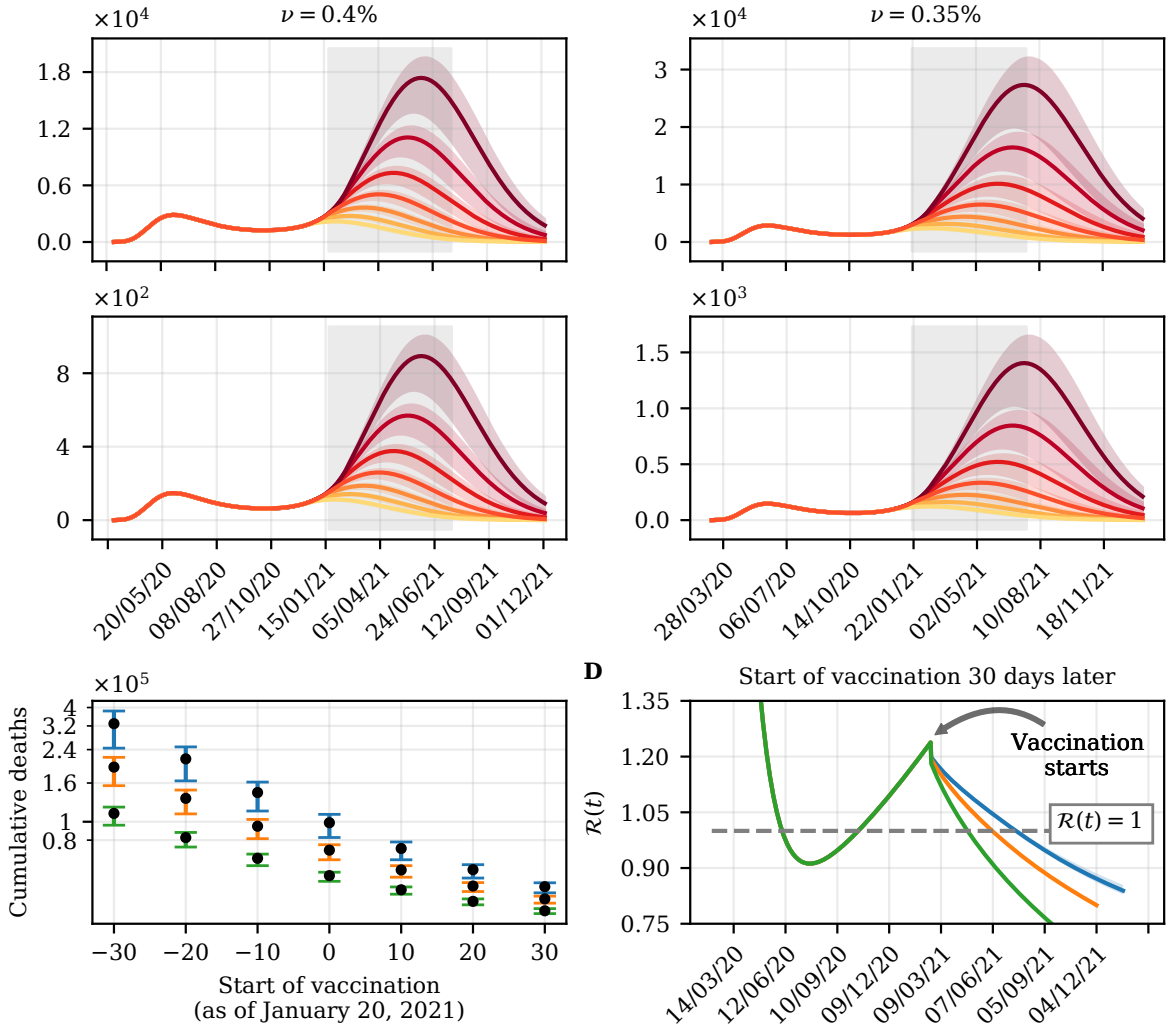

D Start of vaccination 30 days later
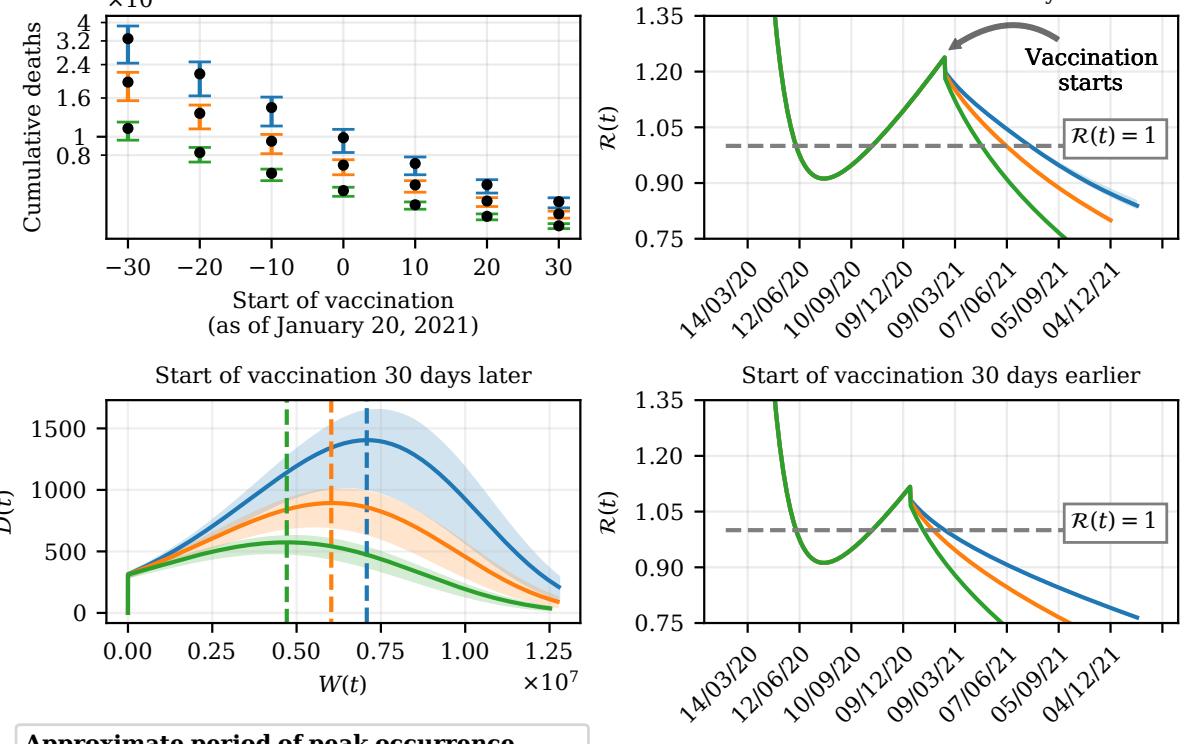

Approximate period of peak occurrence

- - 03/2021 - - 04/2021 - - 07/2021

Figure 3: Importance of rolling out vaccination program as soon as possible. (A) Simulations considering scenarios in which the start of vaccination is advanced or delayed by up to 30 days in relation to the actual start date. The grey shaded area represents the six-month interval from the start of vaccination, January 20 , 2021. (B) Cumulative number of dead and infected individuals when reaching $80 \%$ vaccination coverage, varying the day on which vaccination is started, as well as vaccination rates. The error bars are associated with the $95 \%$ credible interval of the simulations and the black dots refer to the simulations when the maximum a posterior of the inferred parameters are adopted. (C) Relationship between the number of individuals vaccinated and dead over time, given a 30-day early or late start in vaccination in relation to the actual date. The vertical dashed lines express the approximate period at which deaths would peak, for each particular vaccination rate. (D) Effective reproduction number, given the analysed scenarios.

in the first half of October 2021, when simulations indicate that 485 cases (290-629) would be reported daily. In the same period, but assuming a slower vaccination rate, the daily number of confirmed cases would be $2,171(1,280-2,842)$, when $64.09 \%$ of the population would be immunised. In addition, overall low vaccination coverage combined with a lethargic immunisation program could raise the possibility of a resurgence of cases (and hence deaths) as early as 2022. After experiencing a reduction in the number of cases, to a large extent due to vaccination, in February 2022 there would be the smallest number of infected individuals since the onset of the epidemic,
519 (206-856). However, in the following months, the incidence of cases could increase again, reaching 1,188 new cases $(313-2,492)$ per day by mid-May 2022.

Thousands of people have also been missing their second dose of vaccine in RJ, further complicating a campaign already marred by backwardness and supply shortages. To the best of our knowledge, there are still no studies that confirm the overall efficacy of all vaccines used in RJ when only one shot is provided (except for the Janssen vaccine), although some studies have already reported relevant results. ${ }^{17-21}$ In the absence of 
Table 1: Numerical results corresponding to figure 3B. For the scenarios of advance and delay in the start of vaccination, the cumulative number of cases and deaths when the target vaccination coverage is reached are shown.

\begin{tabular}{|c|c|c|c|c|}
\hline & \multicolumn{2}{|c|}{ Vaccination start day } & \multicolumn{2}{|c|}{ When the expected vaccination coverage is reached } \\
\hline & Days & Scenario & Cumulative cases $(95 \% \mathrm{CI})$ & Cumulative deaths $(95 \% \mathrm{CI})$ \\
\hline \multirow{7}{*}{$v=0.35 \%$} & 30 & \multirow{3}{*}{ Delayed } & $6,398,467(4,754,038-7,462,813)$ & $328,781(244,308-383,445)$ \\
\hline & 20 & & $4,174,021(3,195,294-4,827,030)$ & $214,497(164,218-248,034)$ \\
\hline & 10 & & $2,773,525(2,216,328-3,148,266)$ & $142,541(113,904-161,802)$ \\
\hline & \multicolumn{2}{|c|}{ January 20, 2021} & $1,922,585(1,607,030-2,128,694)$ & $98,812(82,596-109,401)$ \\
\hline & 10 & \multirow{3}{*}{ Advanced } & $1,405,981(1,226,504-1,521,840)$ & $72,264(63,041-78,217)$ \\
\hline & 20 & & $1,087,583(982,361-1,155,403)$ & $55,901(50,493-59,386)$ \\
\hline & 30 & & $884,559(820,975-927,468)$ & $45,466(42,198-47,671)$ \\
\hline \multirow{7}{*}{$v=0.40 \%$} & 30 & \multirow{3}{*}{ Delayed } & $3,777,084(3,013,911-4,253,655)$ & $194,101(154,896-218,577)$ \\
\hline & 20 & & $2,584,311(2,139,901-2,859,009)$ & $132,819(109,986-146,928)$ \\
\hline & 10 & & $1,842,755(1,584,306-2,000,994)$ & $94,714(81,424-102,857)$ \\
\hline & \multicolumn{2}{|c|}{ January 20, 2021} & $1,378,382(1,226,446-1,472,044)$ & $70,846(63,037-75,659)$ \\
\hline & 10 & \multirow{3}{*}{ Advanced } & $1,083,992(992,378-1,141,541)$ & $55,716(51,008-58,674)$ \\
\hline & 20 & & $891,948(834,291-929,527)$ & $45,846(42,882-47,777)$ \\
\hline & 30 & & $762,443(724,428-788,777)$ & $39,189(37,236-40,543)$ \\
\hline \multirow{7}{*}{$v=0.50 \%$} & 30 & \multirow{3}{*}{ Delayed } & $2,153,052(1,866,261-2,324,465)$ & $110,653(95,920-119,456)$ \\
\hline & 20 & & $1,605,042(1,431,167-1,708,748)$ & $82,496(73,563-87,822)$ \\
\hline & 10 & & $1,248,913(1,141,622-1,313,404)$ & $64,196(58,673-67,522)$ \\
\hline & \multicolumn{2}{|c|}{ January 20, 2021} & $1,011,948(944,239-1,054,368)$ & $52,013(48,532-54,193)$ \\
\hline & 10 & \multirow{3}{*}{ Advanced } & $851,884(807,515-881,950)$ & $43,786(41,506-45,332)$ \\
\hline & 20 & & $740,340(709,772-762,726)$ & $38,053(36,482-39,204)$ \\
\hline & 30 & & $660,212(637,960-677,854)$ & $33,935(32,791-34,841)$ \\
\hline
\end{tabular}

such information, we assume two scenarios regarding vaccine efficacies when only the first shot is given, that is, efficacies are weakened proportionally to $\mu=25 \%$ and $\mu=50 \%$. Surveys show that around $14.5 \%$ of the Brazilian population somewhat disagree, strongly disagree, or remain neutral regarding vaccination. $^{2}$ Within this context, we also consider scenarios with low $(\alpha=20 \%)$ and moderate $(\alpha=10 \%)$ demand for the second dose of vaccines (when applicable), as well as the best scenario in which $\alpha=0 \%$. Simulations for the number of dead individuals as of the actual day vaccination has started, combining factors associated with parameters $\mu$ and $\alpha$, are shown in figure 4D. Initially, assume that the first dose of vaccines would yield an efficacy proportional to $\mu=25 \%$ of the overall efficacy when both doses are given. In a scenario subject to slow vaccination, $70 \%$ of the eligible population would have been immunised in approximately 211 days. After this time frame, the number of daily deaths would be $357(236-437)$ if $20 \%$ of the population eligible to be vaccinated missed their second dose. If the percentage of individuals who do not receive the second dose dropped to $10 \%$, the death toll would be 263 (174-322) in the same period. In turn, if the efficacy of vaccines was weakened by $\mu=50 \%$ when the second dose is neglected, the number of dead individuals on the same day could be 292 (193-358) and 237 (156-290), bearing in mind the two scenarios related to vaccination coverage with the second dose, respectively. The corresponding numerical results are shown in table 2.

\section{More ambitious vaccination targets and avertable deaths}

We attempt to infer deaths that could have been averted simply by having vaccination started days earlier or if the daily rate of vaccination had been higher. Figure $4 \mathrm{~B}$ shows the relationship between vaccinated individuals and cumulative deaths over time. We simulate the model using the benchmark vaccination rate $(v=0.40 \%)$ and compare the outcomes in the context of a faster vaccination $(v=0 \cdot 50 \%)$, making allowance for different days for the start of vaccination from the day it actually started. Simulations show that presumably not-so-challenging measures, such as having anticipated the vaccination campaign roll-out by just ten days, combined with an average vaccination rate approximately $25 \%$ faster, could have averted 15,129 deaths $(12,029-16,986)$ in relation to the actual scenario; from a more optimistic, yet still realistic, perspective on the vaccina-
280 281 282 283 

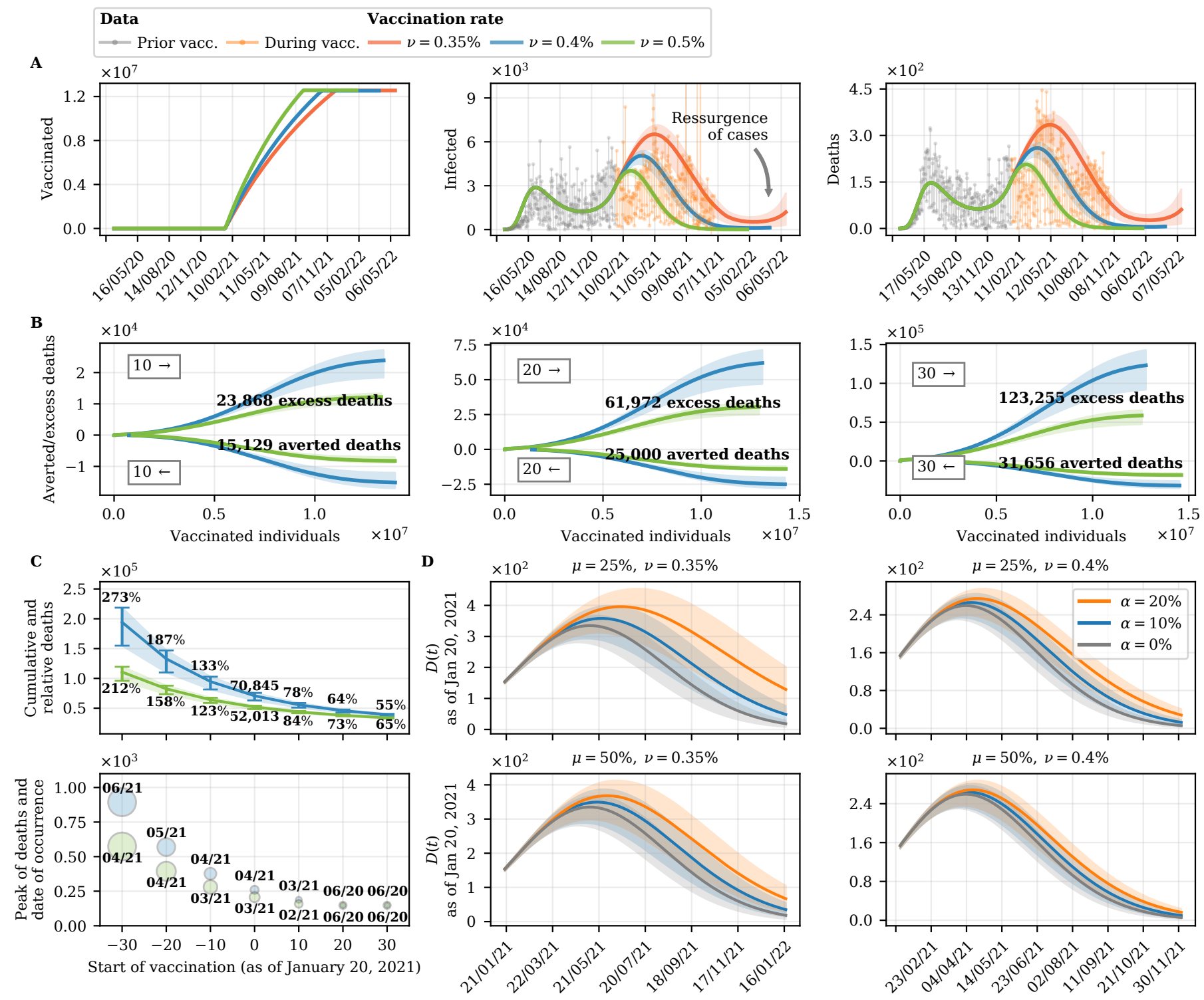

Figure 4: Flawed vaccination policy and excess deaths. (A) Model simulation where part of the population eligible to be vaccinated does not receive any dose. (B) Ratio between the number of deaths given potential scenarios in which the start of vaccination is ahead of the actual date. Scenarios where vaccination would be implemented 10, 20, and 30 days before January 20, 2021 are considered, as well as two vaccination rates $(v=0.40 \%$ and $v=0 \cdot 50 \%)$, and excess deaths are estimated. (C) Variation in the cumulative number of deaths and the number of deaths at the peak of the epidemic curve (during vaccination) taking into account the start of vaccination on different days. The relative percentage amount of cumulative deaths is shown, as well as the month in which deaths would peak. (D) Simulation considering that part of the population proportional to $\alpha$ does not take the second dose of the vaccine. Two scenarios are considered in which only the first dose of the vaccine has efficacy equivalent to $\mu \eta$, combined with two vaccination rates $(v=0 \cdot 35 \%$ and $v=0 \cdot 40 \%)$.

tion roll-out, consider a 30-day advance on the date on which the campaign actually started. In this framework, 31,657 deaths $(25,801-35,117)$ could have been prevented, which represents $44.68 \%$ of the deaths $(40.93 \%-46.41 \%)$ that would have occurred since vaccination was started, assuming a vaccination rate equal to $v=0.40 \%$.

When vaccination became available in RJ, 490,821 cases and 28,215 deaths had already been reported (on that day, there were 4,015 new cases, with 189 deaths). Figure 4C shows that by increasing the vaccination rate, and even under the hy- pothesis of delay, the number of deaths could drop to 110,653 $(95,920-119,456)$ when the target vaccination coverage in October 2021 had been reached. In contrast, starting mass vaccination 30 days before January 20, 2021, when RJ had 457,160 cases $(448,776-467,785)$ and 23,546 deaths $(23,114-24,093)$, could have caused the number of deaths to drop to $20 \%$ compared to the worst-case scenario, assuming that $v=0.40 \%$.

Such delays could also affect the prevalence of the disease, causing the incidence of cases to be longer-lasting at high levels and, consequently, causing the peak of deaths to be shifted 
Table 2: Numerical results corresponding to figure 4D. Cumulative number of deaths and upon reaching $70 \%$ vaccination coverage considering that part of the population $(\alpha)$ does not take the second dose of the vaccine for two scenarios in which only the first dose of the vaccine has efficacy equivalent to $\mu \eta$.

\begin{tabular}{|c|c|c|c|c|c|}
\hline & \multirow[b]{2}{*}{$\mu(\%)$} & \multirow[b]{2}{*}{$\alpha(\%)$} & \multicolumn{2}{|c|}{ Coverage of $70 \%$ of the eligible population } & \multirow[b]{2}{*}{ Cumulative deaths $(95 \% \mathrm{CI})$} \\
\hline & & & $\begin{array}{l}\text { Days to achieve (from } \\
\text { the start of vaccination) }\end{array}$ & Deaths on this day $(95 \% \mathrm{CI})$ & \\
\hline \multirow{5}{*}{$v=0.35 \%$} & \multirow{2}{*}{$0 \cdot 25$} & $0 \cdot 2$ & 211 & $357(236-437)$ & $133,808(103,822-155,110)$ \\
\hline & & $0 \cdot 1$ & 214 & $263(174-322)$ & $112,439(91,105-127,003)$ \\
\hline & \multirow{2}{*}{$0 \cdot 5$} & $0 \cdot 2$ & 213 & $292(193-358)$ & $118,394(94,759-134,842)$ \\
\hline & & $0 \cdot 1$ & 215 & $237(156-290)$ & $107,299(87,930-120,292)$ \\
\hline & \multicolumn{2}{|c|}{$\alpha=0 \cdot 0$} & 217 & $190(125-234)$ & $98,812(82,596-109,401)$ \\
\hline \multirow{5}{*}{$v=0.40 \%$} & \multirow{2}{*}{$0 \cdot 25$} & $0 \cdot 2$ & 185 & $171(122-202)$ & $81,892(70,641-89,077)$ \\
\hline & & $0 \cdot 1$ & 187 & $133(94-156)$ & $75,511(66,331-81,280)$ \\
\hline & \multirow{2}{*}{$0 \cdot 5$} & $0 \cdot 2$ & 187 & $143(102-169)$ & $77,392(67,618-83,575)$ \\
\hline & & $0 \cdot 1$ & 188 & $121(86-143)$ & $73,814(65,126-79,165)$ \\
\hline & \multicolumn{2}{|c|}{$\alpha=0 \cdot 0$} & 190 & $100(71-118)$ & $70,846(63,037-75,659)$ \\
\hline
\end{tabular}

forward. According to simulations, at the worst stage of the epidemic, there could be up to 893 deaths $(706-1,003)$ a day if there had been a 30-day delay in making vaccines available to the population, considering the benchmark vaccination rate, shifting the peak two months ahead of what is expected without such a delay. Figure $4 \mathrm{C}$ supports the fact that delays in the start of the vaccination campaign cause adverse effects that are more severe when the vaccination process is slower.

\section{Discussion}

551 words

Until October 27, 2021, approximately 290 days since the rollout of vaccination in Brazil, RJ was one of the states that had received the most doses per 100,000 inhabitants, about 163,561, as shown in figure 2A. Altogether, Brazil had about 155,727 doses per 100,000 inhabitants. In general, access to vaccines in Brazil was delayed, and this ends up affecting the pace of vaccination even in the states with the most supply of doses. This fact becomes clear when we place the situation in Brazil side by side with that of some other countries, such as Canada, in terms of access to vaccines. Vaccination was launched in Canada on December 14, 2020, nearly one month earlier than in Brazil. In the 290-day window since the launch of the vaccination in Canada, approximately 147,820 doses per 100,000 inhabitants had been administered, a pace similar to what had been performed in Brazil. However, as of October 27, 2021, Canada had reached around 153,480 vaccines administered per 100,000 inhabitants. Considering a seven-day rolling average of daily new deaths, at that time Canada had 0.89 deaths per million people, whereas Brazil had 1.64 deaths. Such statistics shed light on the importance of getting vaccinated as soon as possible.

Social mobility and NPIs are also important factors when analysing the course of vaccination. The engagement of the Brazilian population in such measures has always been below expectations. ${ }^{22} \mathrm{~A}$ very relevant fact is that only $45.5 \%$ of Brazilians say they wear a face mask outside the home. ${ }^{23}$ Our findings show that the possibility of an eventual resurgence of cases in 2022 should not be overlooked, even though most of the population has been vaccinated. This concern even brings up discussions about possible loss of immunity and the need for extra doses, ${ }^{24}$ although vaccines may remain limited, especially in low-incoming countries, ${ }^{25,26}$ making NPIs essential even after achieving adequate vaccine coverage.

The analysed scenarios reflect current knowledge about vaccination in RJ, from the perspective of available data. The persistence of such predictions depends to some extent on the confirmation of the hypotheses put forward. Particularly regarding vaccine hesitancy (whether for both doses or just the second), the inaction of certain people depends a lot on facts that cannot be predicted. Despite this, social network posts can provide insight into attitudes and sentiments towards vaccination, for instance. From December 1, 2020, to March 31, 2021, a lexiconbased sentiment analysis of Twitter posts shows a steady trend in people's perception of Pfizer and Moderna vaccines, while hesitation over the Oxford-AstraZeneca vaccine appears to be increasing over time. ${ }^{27}$ Nevertheless, until October 27, 2021, Oxford-Astrazeneca vaccines supplied most of the Brazilian demand, with $34.8 \%$ of all vaccines distributed so far (see figure $2 \mathrm{~A}$ ). This could be an indication that, even if the vaccine is available, popular sentiment may be volatile enough that eventually people would not return for the second dose or possible additional doses, especially for vaccines where the interval between doses is high. In this context, social networks play a fundamental role in shaping the opinion of part of the population, since the sharing of narratives and personal opinions without scientific background comes to the knowledge of many peo- 
ple. ${ }^{28,29}$ In Brazil, the oscillations regarding the feelings analysed in the posts on social networks are due, in large part, to political actions. ${ }^{30}$

\section{Contributors}

G.B.L., L.A., R.C.C.A., and S.M.C.M. conceived the study; G.B.L. collected the data; All authors analysed the data; G.B.L. designed the models and conducted computations; All authors analysed and discussed the results; G.B.L. wrote the manuscript, with input from all authors; G.B.L. prepared the figures and tables; L.A., R.C.C.A., S.M.C.M., and R.A.M. revised the content critically; R.C.C.A. and S.M.C.M. supervised the study; R.A.M. validated the study; All authors contributed to the final draft.

\section{Declaration of interests}

The authors declare no competing interests.

\section{Data sharing}

Code to replicate analysis and figures supporting the findings of the manuscript are available via the project GitHub repository at https://github.com/gustavolibotte/vaccinesCOVID-19. The code is licensed under the MIT license. Source data are provided with this paper and all data used in this study can be downloaded from the cited sources.

\section{Acknowledgements}

The authors would like to thank the Ministry of Science, Technology, Innovation, and Communication (MCTIC) of Brazil. Gustavo Libotte and Lucas dos Anjos are supported by postdoctoral fellowships from the Carlos Chagas Filho Foundation for Supporting Research in the State of Rio de Janeiro (FAPERJ), grant number E-26/200.347/2021, and from the Institutional Training Program (PCI) of the Brazilian National Council for Scientific and Technological Development (CNPq), grant number 301327/2020-3, respectively.

\section{References}

1 Mathieu E, Ritchie H, Ortiz-Ospina E, et al. A global database of COVID19 vaccinations. Nat Hum Behav 2021; 5: 947-53.

2 Lazarus J V., Ratzan SC, Palayew A, et al. A global survey of potential acceptance of a COVID-19 vaccine. Nat Med 2021; 27: 225-8.

3 Hallal PC, Victora CG. Overcoming Brazil's monumental COVID-19 failure: an urgent call to action. Nat Med 2021; 27: 933-933.
4 Malta M, Vettore MV, Silva CMFP, Silva AB, Strathdee SA. Political neglect of COVID-19 and the public health consequences in Brazil: The high costs of science denial. EClinicalMedicine 2021; 35: 100878 .

5 Fonseca P. Brazil vaccination pace slows as production issues halt second doses. Reuters. 2021. https://www.reuters.com/world/americas/brazilvaccination-pace-slows-production-issues-halt-second-doses-2021-06-01/ (accessed 1 June 2021).

6 Ionova A. Millions in Brazil are missing their second vaccine dose, adding to the burden of a hard-hit nation. The New York Times. 2021. https://www.nytimes.com/2021/06/27/world/brazil-covid-vaccine.html (accessed 27 June 2021).

7 Fonseca P, Spring J, Reese C. Brazil to pause production of AstraZeneca vaccine due to lack of ingredients. Reuters. 2021. https://www.reuters.com/world/brazil-pause-production-astrazenecavaccine-due-lack-ingredients-2021-05-13/ (accessed 27 June 2021).

8 Reuters Staff. Rio de Janeiro halts COVID-19 shots as vaccine supplies dry up. Reuters. 2021. https://www.reuters.com/article/health-coronavirus-riovaccines-idUSL1N2KL12Q (accessed 14 July 2021).

9 Phillips T. 'We're being left behind': anger and confusion in Brazil as vaccine program lags. the Guardian. 2021. https://www.theguardian.com/world/2020/dec/31/brazil-coronavirusvaccine-jair-bolsonaro (accessed 15 July 2021).

10 Barberia LG, Costa SF, Sabino EC. Brazil needs a coordinated and cooperative approach to tackle COVID-19. Nat Med 2021; 27: 1133-4.

11 Gallotti R, Valle F, Castaldo N, Sacco P, De Domenico M. Assessing the risks of 'infodemics' in response to COVID-19 epidemics. Nat Hum Behav 2020; 4: 1285-93.

12 Cota W. Monitoring the number of COVID-19 cases and deaths in Brazil at municipal federative units level. Scielo Prepr 2020. DOI: $10.1590 /$ SciELOPreprints.362

13 Libotte GB, Anjos L, Almeida RCC, Malta SMC, Silva RS. Framework for enhancing the estimation of model parameters for data with a high level of uncertainty. medRxiv 2020. DOI:10.1101/2020.12.17.20248389.

14 Rasmussen CE, Williams CKI. Gaussian Processes for Machine Learning. The MIT Press, Cambridge, 2005.

15 Ching J, Chen Y-C. Transitional Markov Chain Monte Carlo Method for Bayesian Model Updating, Model Class Selection, and Model Averaging. J Eng Mech 2007; 133: 816-32.

16 Brazilian Institute of Geography and Statistics. Population projection (in Portuguese). IBGE. 2021. https://www.ibge.gov.br/apps/populacao/projecao/index.html (accessed 12 September 2021).

17 Vasileiou E, Simpson CR, Shi T, et al. Interim findings from first-dose mass COVID-19 vaccination roll-out and COVID-19 hospital admissions in Scotland: a national prospective cohort study. Lancet 2021; 397: 1646-57.

18 Lopez Bernal J, Andrews N, Gower C, et al. Effectiveness of the PfizerBioNTech and Oxford-AstraZeneca vaccines on covid-19 related symptoms, hospital admissions, and mortality in older adults in England: test negative case-control study. BMJ 2021; 373: n1088.

19 Chodick G, Tene L, Patalon T, et al. Assessment of effectiveness of 1 dose of BNT162B2 vaccine for SARS-CoV-2 infection 13 to 24 days after immunization. JAMA Netw Open 2021.

20 Shrotri M, Krutikov M, Palmer T, et al. Vaccine effectiveness of the first dose of ChAdOx1 nCoV-19 and BNT162b2 against SARS-CoV-2 infection in residents of long-term care facilities in England (VIVALDI): a prospective cohort study. Lancet Infect Dis 2021; 21: 1529-38.

21 Hyams C, Marlow R, Maseko Z, et al. Effectiveness of BNT162b2 and ChAdOx 1 nCoV-19 COVID-19 vaccination at preventing hospitalisations in people aged at least 80 years: a test-negative, case-control study. Lancet Infect Dis 2021; 21: 1539-48. 
22 Dantas RCC, Campos PA, Rossi I, Ribas RM. Implications of social distancing in Brazil in the COVID-19 pandemic. Infect Control Hosp Epidemiol 2020; 1-2.

23 Villela EFM, López RVM, Sato APS, et al. COVID-19 outbreak in Brazil: adherence to national preventive measures and impact on people's lives, an online survey. BMC Public Health 2021.

24 Callaway E. COVID vaccine boosters: the most important questions. Nature 2021 ; 596: 178-80.

25 Maxmen A. COVID boosters for wealthy nations spark outrage. Nature 2021; published online July 30. DOI:10.1038/d41586-021-02109-1.

26 Josephson A, Kilic T, Michler JD. Socioeconomic impacts of COVID-19 in low-income countries. Nat Hum Behav 2021; 5: 557-65.

27 Marcec R, Likic R. Using Twitter for sentiment analysis towards AstraZeneca/Oxford, Pfizer/BioNTech and Moderna COVID-19 vaccines. Postgrad Med J 2021; 0: 1-7.

28 Johnson NF, Velásquez N, Restrepo NJ, et al. The online competition between pro- and anti-vaccination views. Nature 2020; 582: 230-3.

29 Paul E, Steptoe A, Fancourt D. Attitudes towards vaccines and intention to vaccinate against COVID-19: Implications for public health communications. Lancet Reg Heal - Eur 2021; 1: 100012.

30 Garcia K, Berton L. Topic detection and sentiment analysis in Twitter content related to COVID-19 from Brazil and the USA. Appl Soft Comput 2021; 101: 107057 
Figures

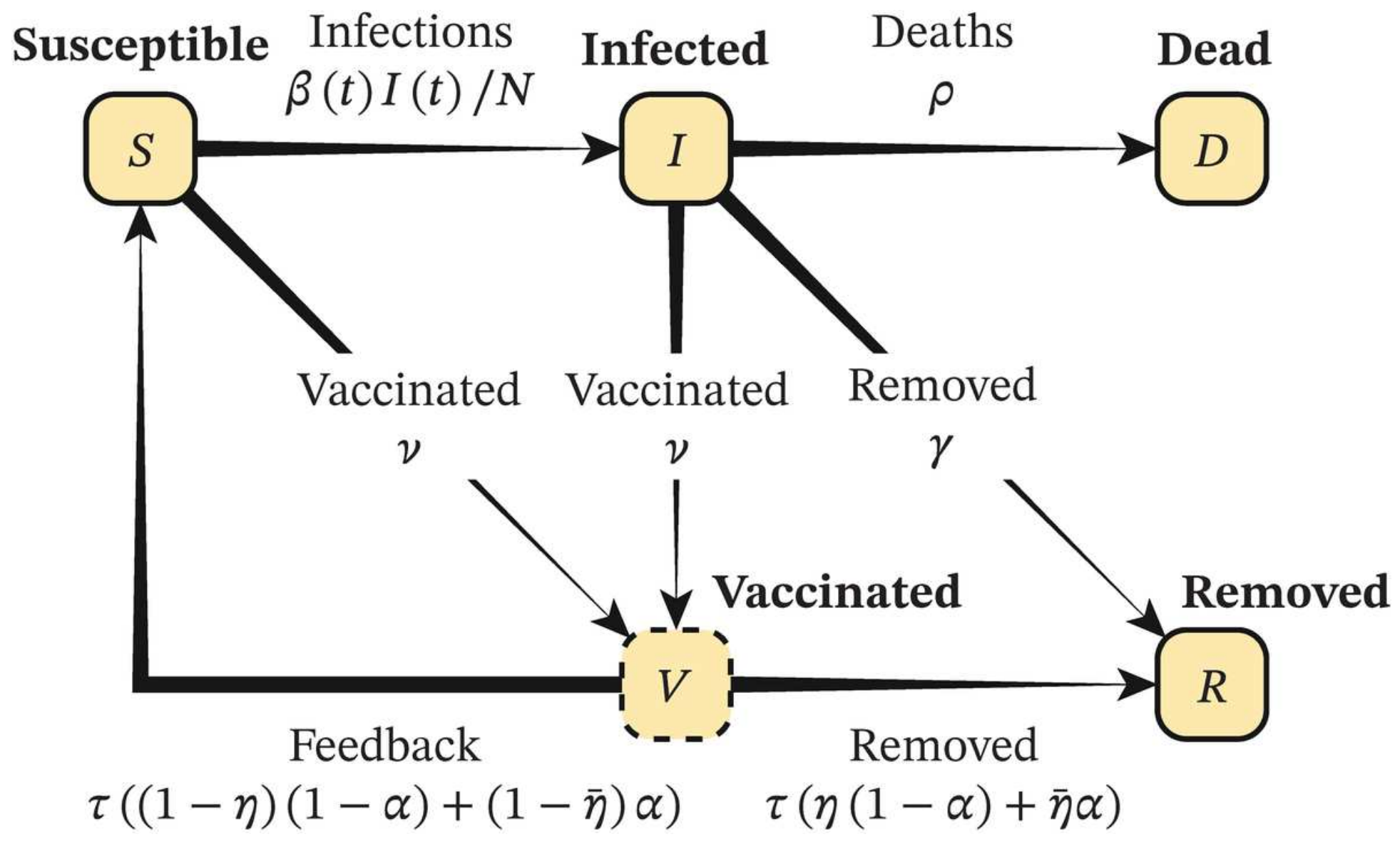

Figure 1

Schematic representation of the model. 
A

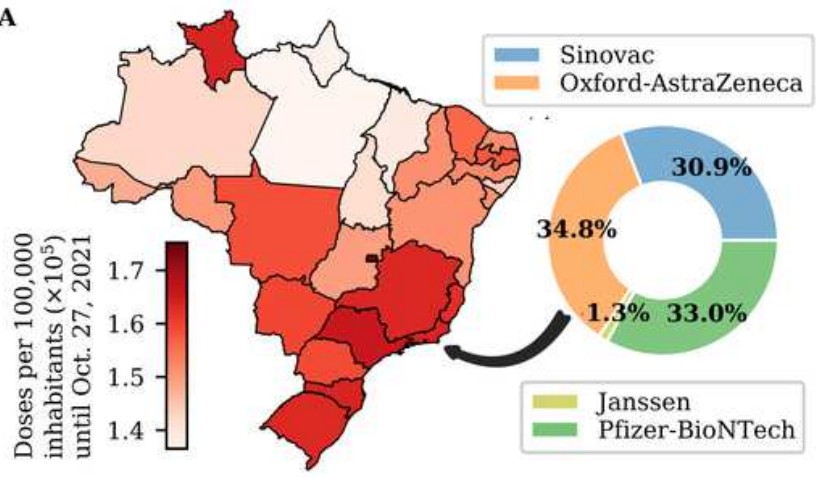

B

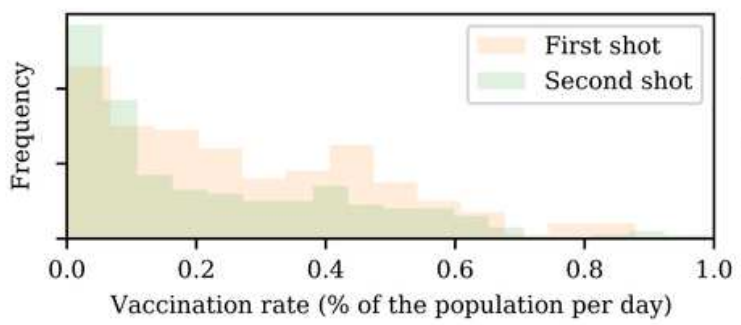

C

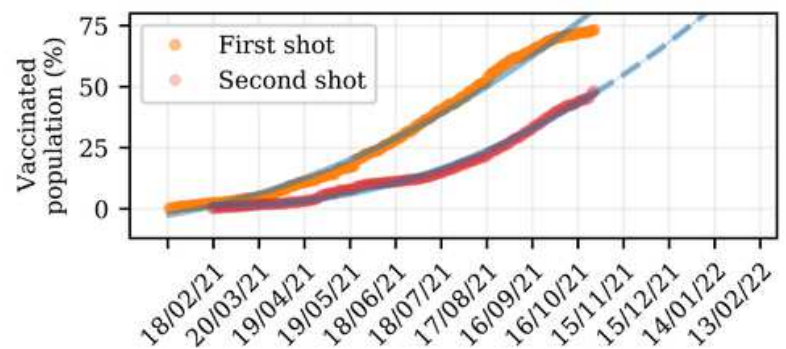

D

Data Vaccination rate

$\longrightarrow$ Prior vacc. - During vacc. - $\nu=0.35 \%-\nu=0.4 \%-\nu=0.5 \%$
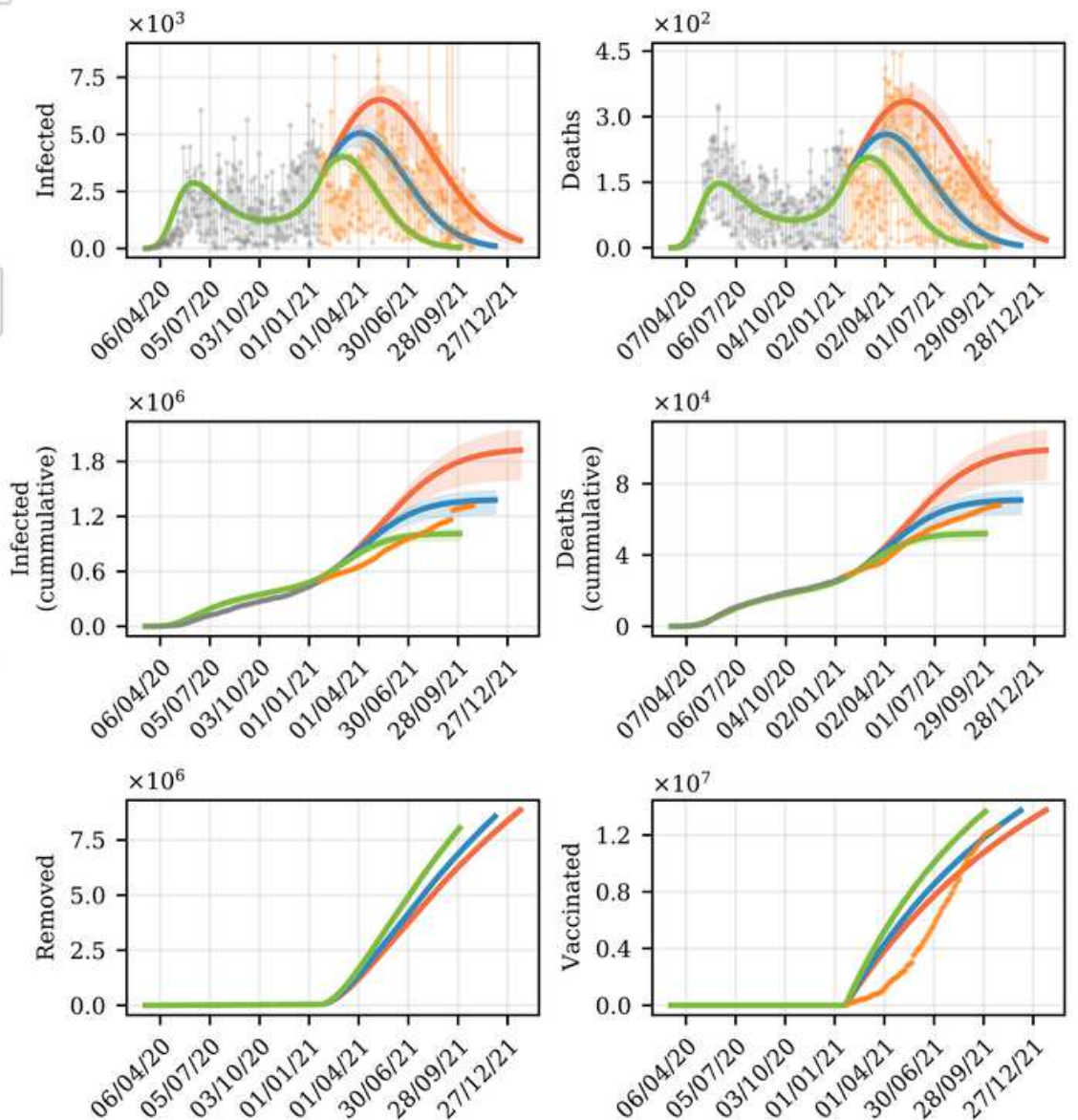

Figure 2

Speed in vaccination and expectation of disease mitigation in RJ. (A) Map of vaccine doses distributed in each Brazilian state per 100,000 inhabitants, and the portion of each type of vaccine destined for RJ, until October 27, 2021. (B) Frequency of vaccination rate for each shot, in terms of percentage of population per day. (C) Perspective of coverage of the population eligible for vaccination for each shot. (D) Model simulation considering different vaccination rates, slow $(v=0 \cdot 35 \%)$, intermediate $(v=0 \cdot 40 \%)$, and fast $(v$ $=0.50 \%)$, taking into account the frequencies $(B)$ and the proportion of vaccines of each type (A). The model is simulated until reaching the same amount of vaccines administered in each scenario (see the lower right frame). The shaded areas represent the $95 \%$ credible interval. Note that the period of the end of vaccination in the scenario where an intermediate vaccination rate is adopted agrees with the prognosis in $(\mathrm{C})$. 

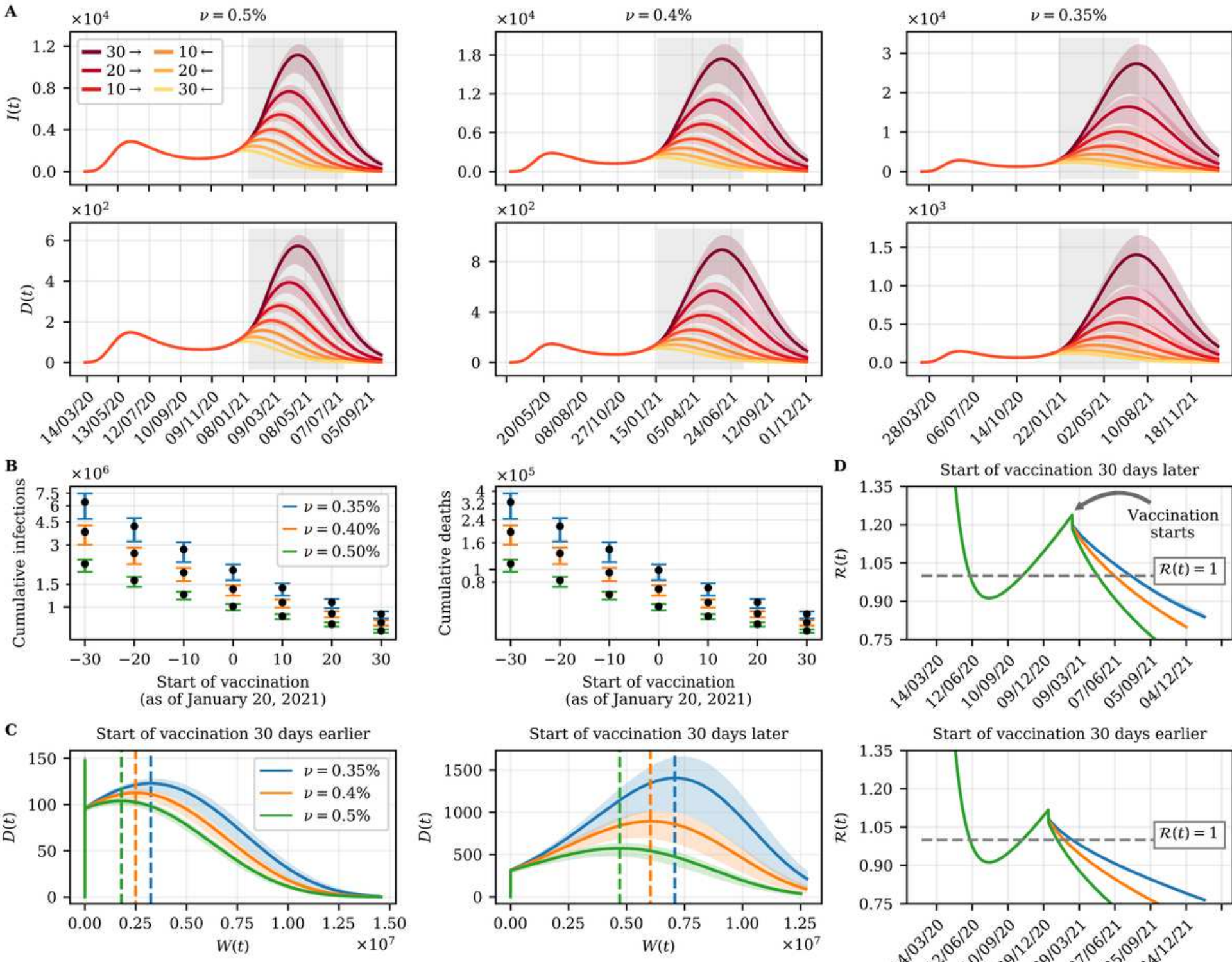

Approximate period of peak occurrence $--01 / 2021 \quad--01 / 2021 \quad--03 / 2021$

\section{Approximate period of peak occurrence} $--03 / 2021 \quad--04 / 2021 \quad-=07 / 2021$
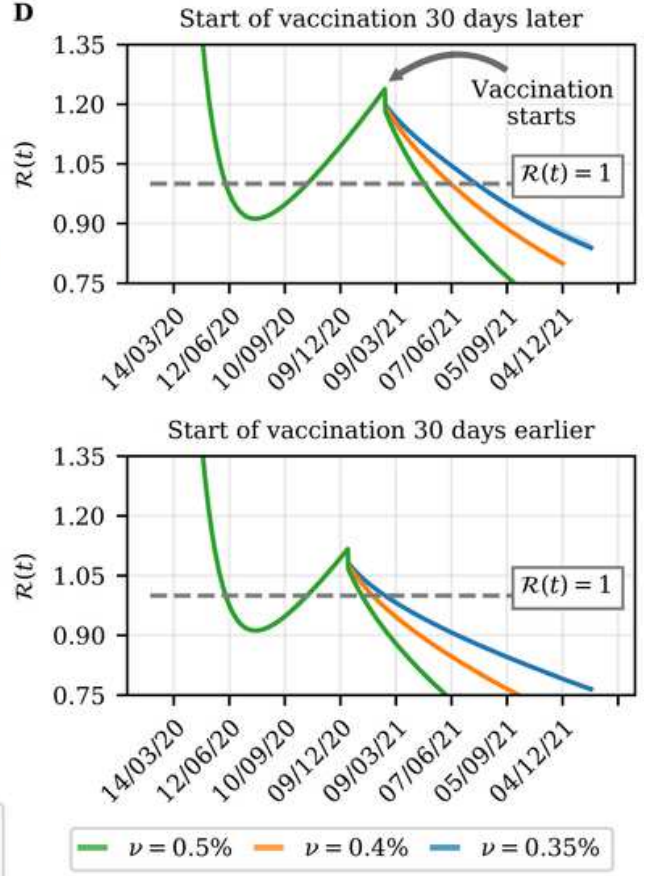

\section{Figure 3}

Importance of rolling out vaccination program as soon as possible. (A) Simulations considering scenarios in which the start of vaccination is advanced or delayed by up to 30 days in relation to the actual start date. The grey shaded area represents the six-month interval from the start of vaccination, January 20,2021 . (B) Cumulative number of dead and infected individuals when reaching $80 \%$ vaccination coverage, varying the day on which vaccination is started, as well as vaccination rates. The error bars are associated with the $95 \%$ credible interval of the simulations and the black dots refer to the simulations when the maximum a posterior of the inferred parameters are adopted. (C) Relationship between the number of individuals vaccinated and dead over time, given a 30-day early or late start in vaccination in relation to the actual date. The vertical dashed lines express the approximate period at which deaths would peak, for each particular vaccination rate. (D) Effective reproduction number, given the analysed scenarios. 


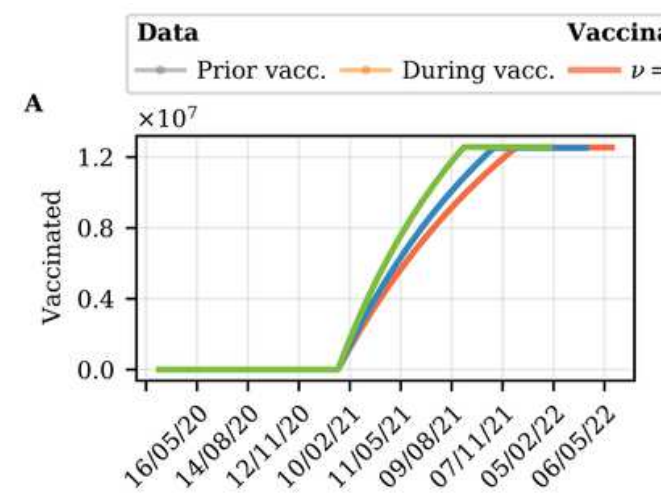

$0.35 \%-\nu=0.4 \%-\nu=0.5 \%$
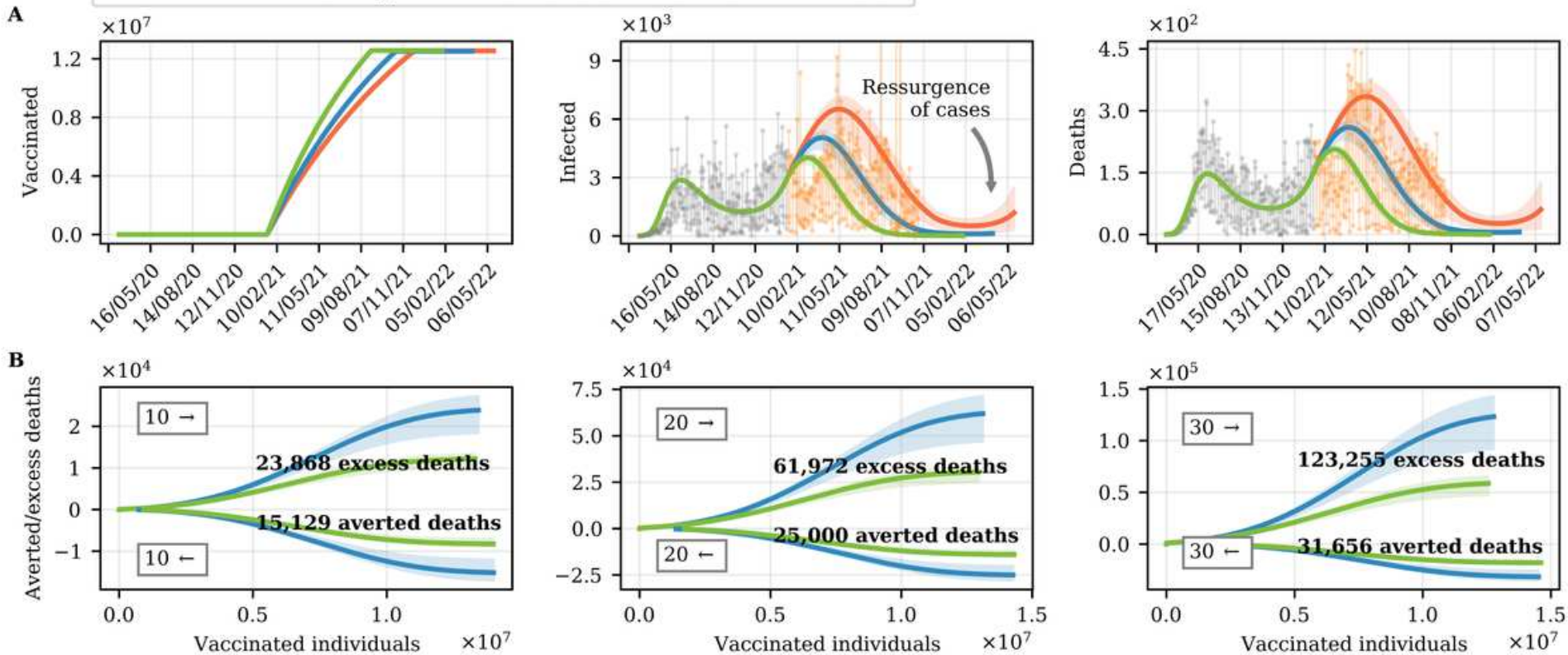

C $\times 10^{5} \quad$ D
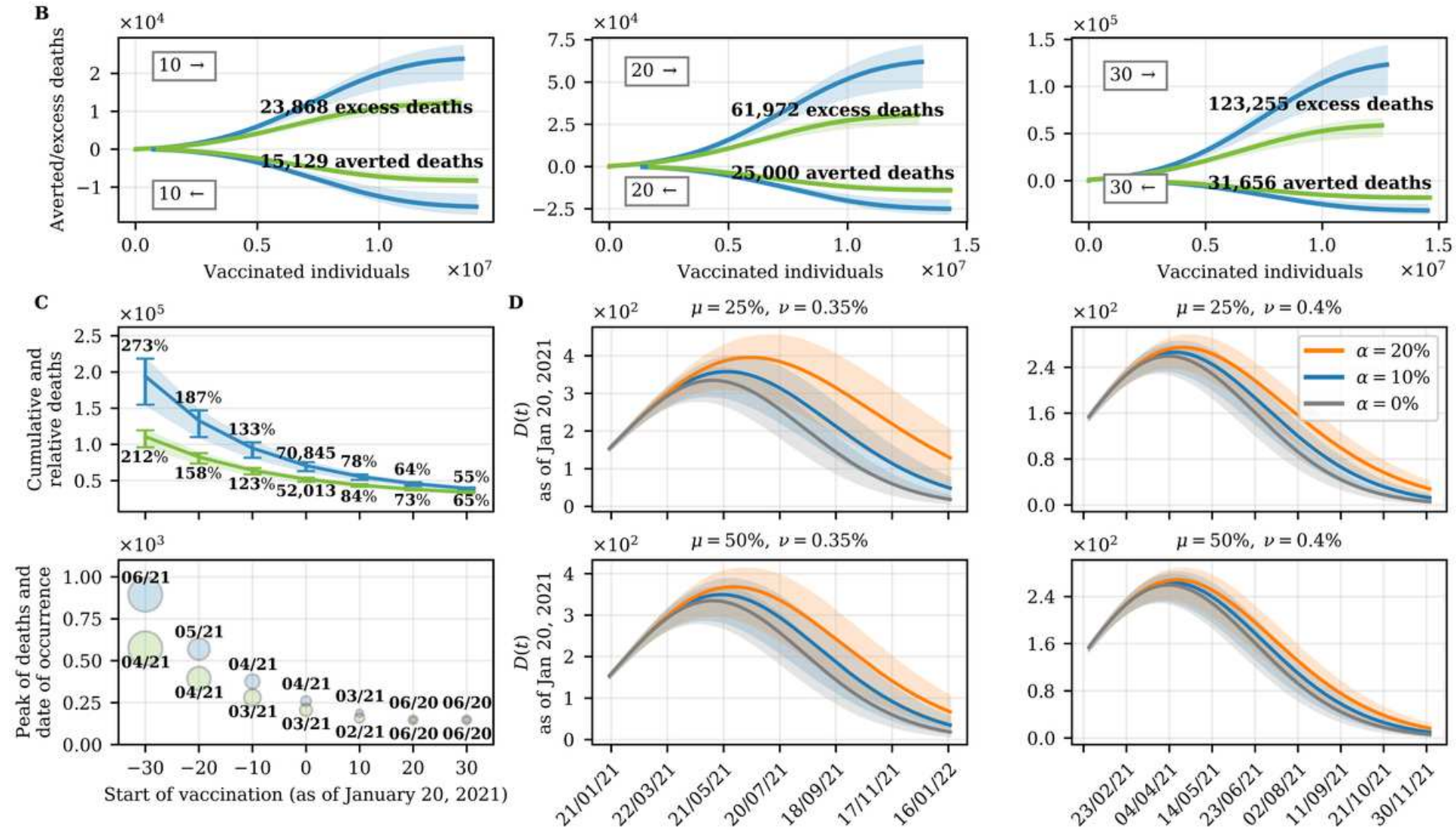

\section{Figure 4}

Flawed vaccination policy and excess deaths. (A) Model simulation where part of the population eligible to be vaccinated does not receive any dose. (B) Ratio between the number of deaths given potential scenarios in which the start of vaccination is ahead of the actual date. Scenarios where vaccination would be implemented 10, 20, and 30 days before January 20, 2021 are considered, as well as two vaccination rates $(v=0.40 \%$ and $v=0.50 \%)$, and excess deaths are estimated. $(C)$ Variation in the cumulative number of deaths and the number of deaths at the peak of the epidemic curve (during vaccination) taking into account the start of vaccination on different days. The relative percentage amount of cumulative deaths is shown, as well as the month in which deaths would peak. (D) Simulation considering that part of the population proportional to a does not take the second dose of the vaccine. Two scenarios are considered in which only the first dose of the vaccine has efficacy equivalent to $\mu \eta$, combined with two vaccination rates $(v=0 \cdot 35 \%$ and $v=0 \cdot 40 \%)$. 


\section{Supplementary Files}

This is a list of supplementary files associated with this preprint. Click to download.

- supplementarymaterial.pdf 